\title{
Electrochemical Desorption of Binary Self-Assembled Monolayers of Butyl Hexadecyl Disulfide on Au(111) Electrodes
}

\author{
Hiroaki AzEHARA, ${ }^{a}$ Soichiro YOSHIMOTO, ${ }^{\mathrm{b}}$ Hirofumi HOKARI, ${ }^{\mathrm{a}, \mathrm{c}}$ Uichi AKIBA, ${ }^{\mathrm{a}}$ \\ Isao TANIGUCHI, ${ }^{b}$ and Masamichi FUJIHIRA*a
}

\begin{abstract}
aDepartment of Biomolecular Engineering, Tokyo Institute of Technology (4259 Nagatsuta, Midori-ku, Yokohama 226-8501, Japan)

${ }^{\mathrm{b}}$ Department of Applied Chemistry and Biochemistry, Faculty of Engineering, Kumamoto University (Kurokami 2-39-1, Kumamoto 860-8555, Japan)

'Present address: CREST, Japan Science and Technology Corporation (Wako, Saitama 351-0198, Japan)
\end{abstract}

Received June 15, 1999 ; Accepted August 30, 1999

\begin{abstract}
Reductive desorption of self-assembled monolayers (SAMs) on Au(111) electrodes prepared in butyl hexadecyl disulfide solutions was studied by cyclic voltammetry. It was shown that behavior of the reductive desorption strongly depended on the concentrations of the disulfide solution. It was concluded from this observation that homogeneously mixed binary SAMs were formed at the high concentration of the asymmetrical disulfide, while the phase-separated mixed SAMs were formed at the low concentration.
\end{abstract}

Key Words : Asymmetrical Disulfides, Binary Self-Assembled Monolayers, Electrochemical Desorption, Au(111)

\section{Introduction}

It is known that organosulfur compounds are useful in modifying electrodes ${ }^{1)}$ because the sulfur moieties of the modifiers can be spontaneously chemisorbed on gold surfaces and form organic monolayers that are termed self-assembled monolayers (SAMs)., ${ }^{2,3)}$ Asymmetrical disulfides have two different kinds of chains linked by two sulfur atoms, which act as adhesives to gold surfaces. ${ }^{2)}$ The use of asymmetrical disulfides has been thought to be a convenient way to obtain SAMs consisting of equal amounts of two different components. ${ }^{4}$ However, surface diffusion of thiolates in SAMs on gold can occur as demonstrated by Stranick et al.$^{5)}$ and disulfide can dissociate in SAMs to form thiolates. ${ }^{6,7)}$ Therefore, the use of asymmetrical disulfides does not always guarantee that two components of the disulfide will mix homogeneously in molecular dimensions. Phaseseparated domains were actually observed by scanning tunneling microscopy (STM) in a SAM made from a solution of disulfide containing hydrocarbon and fluorocarbon chains, although it has not been clarified yet whether the phase separation proceeded during the SAM formation or during the STM measurements. ${ }^{8)}$ For STM under ultra high vacuum, we needed at least $c a .24 \mathrm{~h}$ including evacuation time. In this regard, it is important to develop a new rapid method to investigate the structure of a SAM immediately after preparation.

Since reductive desorption of SAMs were investigated by Widrig et al ${ }^{9)}$ many attempts to characterize SAMs by means of cyclic voltammetry have been made. ${ }^{10-12)}$ The potentials, at which the gold-bound $n$-alkanethiolates were reductively desorbed, were found to shift to more negative potentials with an increase in the number of the carbon atoms of the $n$-alkanethiolates. ${ }^{9)}$ For binary SAMs made from solutions of methyl- and carboxyl-terminated alkanethiols with almost the same chain lengths, i.e. 1-decanethiol and 11-mercaptoundecanoic acid, respectively, only one desorption peak in the negative scan was observed. ${ }^{10)}$ The peak potential of this mixed SAMs, however, depended on the ratio of one component to the other. Contrary, other binary SAMs formed from solutions of 1-hexadecanethiol and 3mercaptopropionic acid exhibited two distinct reductive desorption peaks, $c a .0 .45 \mathrm{~V}$ apart. ${ }^{1,12)}$ It was concluded from observation of the same mixed SAMs using STM in air that these two peaks reflected the presence of two phase-separated domains. ${ }^{12)}$ The exchange reaction between chemisorbed thiolate in a SAM and thiol or disulfide in solution was also reported. ${ }^{13)}$

In the present work, cyclic voltammetry was utilized as a rapid method immediately after preparation of SAMs to investigate whether an asymmetrical disulfide containing two alkyl chains with large difference in their chain lengths forms the homogeneous mixed SAM or not (Fig. 1). It was studied how the

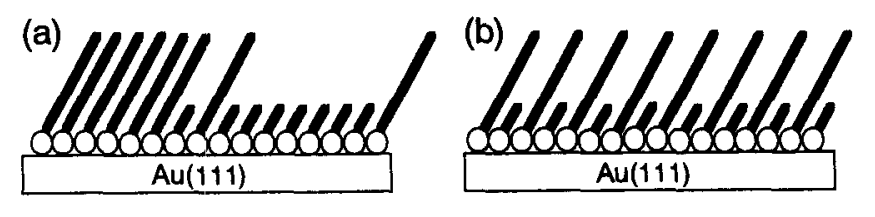

Fig. 1 Schematic illustrations of the SAMs derived from $\mathrm{H} 4 \mathrm{H} 16$ in which (a) phase-separated domains are appeared and (b) butyl and hexadecyl chains are mixed homogeneously in molecular dimensions. 
disulfide concentration in solution for SAM formation affects the structure of the resulting SAMs. It was also studied whether the peak potentials for the reductive desorption really depend on the compositions in the SAMs when they are mixed homogeneously. To answer these questions, SAMs of butyl hexadecyl disulfide $\left[\mathrm{C}_{4} \mathrm{H}_{9}-\mathrm{S}-\mathrm{S}-\mathrm{C}_{16} \mathrm{H}_{33} ; \mathrm{H} 4 \mathrm{H} 16\right]$ were prepared on $\mathrm{Au}(111)$ electrodes at two different concentrations.

\section{Experimental}

$\mathrm{H} 4 \mathrm{H} 16$ as well as dihexadecyl disulfide (H16H16) was synthesized from the corresponding thiols by iodine oxidation. ${ }^{4)}$ These disulfides were purified by high performance liquid chromatography. Monocrystalline gold beads were prepared by melting a gold wire in an $\mathrm{H}_{2}+\mathrm{O}_{2}$ flame. After orientation of the bead was aligned while observing a facet on the bead, they were cut, polished, and annealed in order to expose an $\mathrm{Au}(111)$ surface. ${ }^{14,15)}$ SAMs were obtained by immersing the single crystals into ethanol solutions of disulfides for different periods of time and at two different concentrations ( 10 or $100 \mu \mathrm{M}: \mathrm{M}=\mathrm{mol} \mathrm{dm^{-3 }}$ ) at room temperature. The modified single crystal was used as a working electrode for cyclic voltammetry using a hanging meniscus technique. Scans were initiated at $-200 \mathrm{mV}$ vs. $\mathrm{Ag} / \mathrm{AgCl}$ at a scanning rate of -50 $\mathrm{mV} \mathrm{s}{ }^{-1}$. All measurements were carried out at room temperature under a nitrogen atmosphere within 20 min after the monolayer formation.

\section{Results and Discussion}

In Fig. 2 is shown cyclic voltammograms (CVs) for the SAMs prepared in the solution of $\mathrm{H} 4 \mathrm{H} 16$ at the high concentration $(100 \mu \mathrm{M})$. The CVs for the SAMs formed by dipping for the different periods of time, i.e. $5 \mathrm{~s}, 5 \mathrm{~min}$, and $13 \mathrm{~h}$, were almost the same in their shapes in the cathodic and the following anodic waves. From this observation, we draw the following two conclusions. One is that the monolayer formation was almost completed within $5 \mathrm{~s}$ by immersing a gold electrode in the $100 \mu \mathrm{M}$ solution. The other is that morphology of the resulting monolayer was independent of immersion time in the solution. Thus, such CVs as shown in Fig. 2 can be regarded as CVs of the SAMs where the butyl and hexadecyl chains of the equimolar amount are mixed homogeneously in molecular dimensions.

On the other hand, CVs for the SAMs prepared in the dilute solution of $\mathrm{H} 4 \mathrm{H} 16(10 \mu \mathrm{M})$ shown in Fig. 3 are clearly different from one another in their shapes. It was obvious from a comparison between the CVs in Fig. 3 and those in Fig. 2 that the monolayer formation in this dilute solution was relatively slow and morphology of the SAM changed with the immersion time even after $1 \mathrm{~h}$. Two distinct peaks appeared in the cathodic waves in Figs. 3(g) and 3(h). This observation suggests that, in the SAM prepared from this dilute solution, the phase rich in butane species was separated from that rich in hexadecane ones.
Imabayashi et al. ${ }^{11)}$ previously reported that a less negative peak in a $\mathrm{CV}$ for a phase-separated binary SAM disappeared after multiple potential cycling in a potential range including the less negative peak

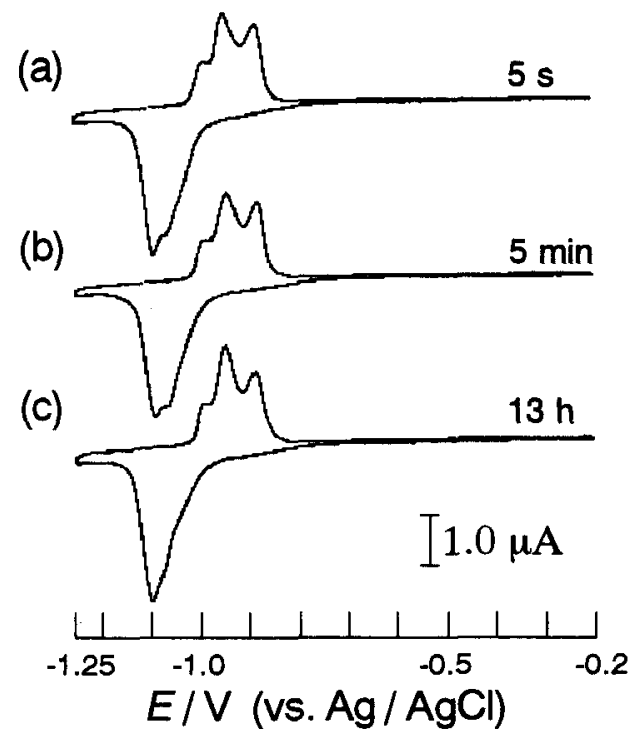

Fig. $2 \mathrm{CVs}$ of the SAMs formed in a $100 \mu \mathrm{M}$ solution of H4H16 for different periods of immersion time: (a) $5 \mathrm{~s}$; (b) $5 \mathrm{~min}$; and (c) $13 \mathrm{~h}$.

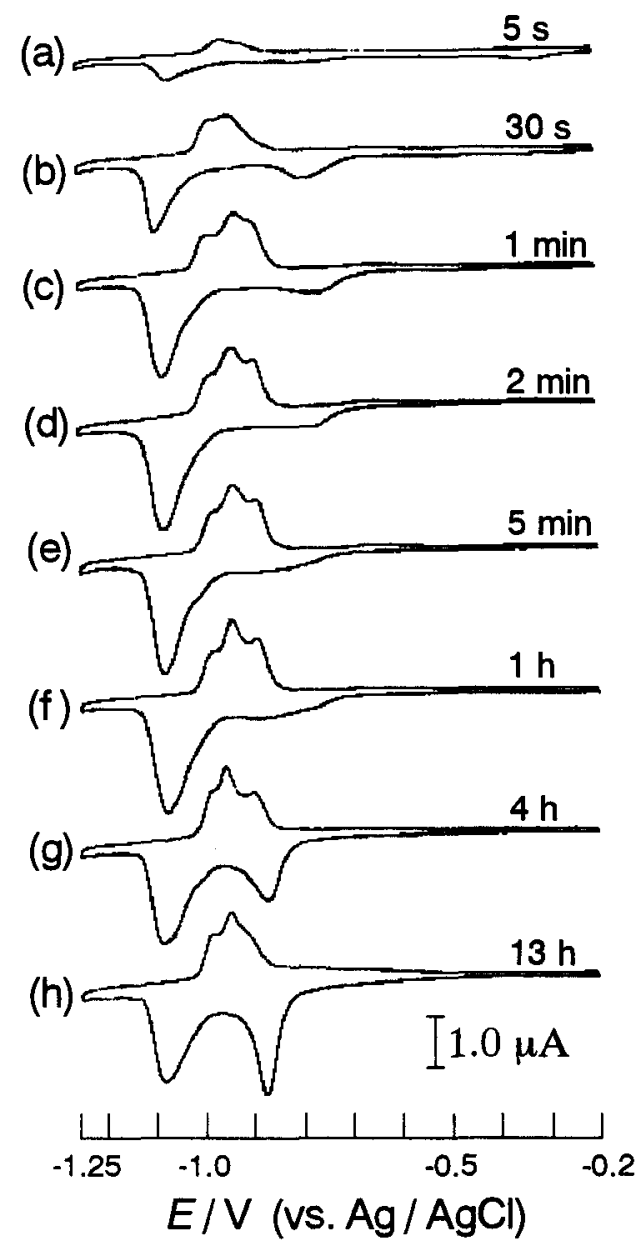

Fig. $3 \mathrm{CVs}$ of the SAMs formed in a $10 \mu \mathrm{M}$ solution of $\mathrm{H} 4 \mathrm{H} 16$ for different periods of immersion time: (a) $5 \mathrm{~s}$; (b) $30 \mathrm{~s}$; (c) $1 \mathrm{~min}$; (d) $2 \mathrm{~min}$; (e) $5 \mathrm{~min}$; (f) $1 \mathrm{~h}$; (g) $4 \mathrm{~h}$; and (h) $13 \mathrm{~h}$. 
potential. We also observed that the first desorption peak at $c a .-0.9 \mathrm{~V}$ vs. $\mathrm{Ag} / \mathrm{AgCl}$ in Fig. 3(h) disappeared completely after multiple scans between -200 and $-970 \mathrm{mV}$ vs. $\mathrm{Ag} / \mathrm{AgCl}$. Thus, this voltammetric feature of the SAM formed in the dilute solution supports further that the phase separation proceeded in the long immersion time.

The most plausible factor causing the difference in observed time dependence of the morphological changes can be the difference in mobility of chemisorbed species between the SAMs during preparation under the two different concentrations. It may safely be concluded from the CVs in Fig. 2 that closely packed SAMs were formed within $5 \mathrm{~s}$ at the high concentration and thus mobility of species in the SAMs during preparation was low. Contrary, as is clear from time dependence of CVs in Fig. 3, it seems that it took long time $(>13 \mathrm{~h})$ to form the closely packed SAMs and thus mobility of species in the SAM under the low concentration can be higher than that in the closely packed SAM. The change in the surface concentration of SAMs prepared from solutions containing dialkyl disulfides or alkanethiols as a function of immersion time was studied by ellipsometry and contact angle measurements. ${ }^{13)}$ The change in packing densities and morphological changes in SAMs of alkanethiols was also studied in detail using STM as a function of reaction time in solution ${ }^{16)}$ and in a gas phase. ${ }^{17)}$ These results support the above interpretation of the present observation in terms of the change in mobility of species in the SAMs. The more facile exchange reaction between the surface and the solution species under the low concentration cannot be ruled out as a cause for the phase separation, although the rate of replacement of the surface species with the solution species of disulfides was much lower than that of thiols. ${ }^{13)}$

Figure 3 shows that the first peak shifted to negative potentials with an increase in the immersion time. It should be noted that formation of the closely packed SAM was not completed in immersion times less than $13 \mathrm{~h}$ in the dilute solution. Therefore, the negative shift of the first peak can be attributed to the increase in the packing density in butane rich domains with the increase in the immersion time. The increase in the density of thiolates in the butane rich phase with the increase in the immersion time is also clear from the simultaneous increase in the charge of desorption of the first peak. The densely packed molecules were stabilized by their lateral interaction between the chains and thus the reduction peak potential shifted to the more negative potentials.

It is surprising that the second peak in the negative scan in Figs. 3(b)-3(h) was observed at ca. $-1080 \mathrm{mV}$ vs. $\mathrm{Ag} / \mathrm{AgCl}$ without negative shifts. In Figs. 3(c)-3(h), almost the same charge for the second peak was observed in contrast with those for the first peak. More surprisingly, the constant second peak potential was almost the same potential as that of the single desorption peak observed in Fig. 2. As described above, the single desorption peak in Fig. 2 was assigned to desorption of the $1: 1$ homogeneous mixture of long and short alkyl chains, while the second peak in Figs. 3(g) and 3(h) was assigned to desorption of molecules in the phase-separated domains rich in hexadecane species. Therefore, if the potential of the second peak were determined only by the composition in the hexadecane rich phase, the second peak potential would be more negative than the peak potential in Fig. 2.

The reason for the above constant potential of the second peak has not been clarified yet. A possible explanation for almost the same potential for the 1 : 1 mixture in Fig. 2 and the phase-separated domains rich in hexadecane species is that density of the former was higher than that of the latter even after $13 \mathrm{~h}$. A second, and perhaps more likely, explanation is that the structure of the hexadecane rich domains is changed into a more easily reducible form by preceding desorption of the butane rich domains during the negative scan. Namely, the edges of the hexadecane rich domains are exposed by the desorption of the butane rich domains and thus the electrochemical reductive desorption was accelerated. Thus, this structural change may cancel the tendency of negative shift of the second peak due to the riches of hexadecane species. The same is true for the negative shift of the desorption peak of the butane rich domains due to protection of them by the surrounding walls of the hexadecane rich domains in addition to the effect of mixing of hexadecane species in them. It should be noted here that the second peak potential in Figs. 3(g) and 3(h) was found to be positive by $c a .50$ $\mathrm{mV}$ compared with the desorption peak potential of the single component SAM prepared from solutions containing only $\mathrm{H} 16 \mathrm{H} 16$.

\section{Conclusion}

The SAMs prepared from solutions containing an asymmetrical disulfide, i.e. $\mathrm{H} 4 \mathrm{H} 16$, at different two concentrations exhibited different $\mathrm{CV}$ characteristics. The CVs suggest that the homogeneous mixed SAM was formed at $100 \mu \mathrm{M}$, while the phase separation was caused at $10 \mu \mathrm{M}$. The difference in the time change in CVs between the SAMs prepared at the two different concentrations was attributed to the difference in mobility of the surface species between the SAMs due to different packing densities. It was also found that the desorption peak potentials did not necessarily depend on composition of the binary mixed SAMs, but might depend on the packing density and the presence of the exposed edges.

\section{Acknowledgment}

This work was supported by a Grant-in-Aid for Priority Area "Electrochemistry of Ordered Interfaces" No. 09237101 from the Ministry of Education, Science, Culture and Sports, Japan. 


\section{References}

1) I. Taniguchi, K. Toyosawa, H. Yamaguchi, and K. Yasukouchi, J. Chem. Soc., Chem. Commun., 1982, 1032 (1982).

2) R. G. Nuzzo and D. L. Allara, J. Am. Chem. Soc., 105, 4481 (1983).

3) A. Ulman, Chem. Rev., 96, 1533 (1996).

4) C. D. Bain, H. A. Biebuyck, and G. M. Whitesides, Langmuir, 5, 723 (1989).

5) S. J. Stranick, A. N. Parikh, Y.-T. Tao, D. L. Allara, and P. S. Weiss, J. Phys. Chem., 98, 7636 (1994).

6) T. Ishida, S. Yamamoto, W. Mizutani, M. Motomatsu, H. Tokumoto, H. Hokari, H. Azehara, and M. Fujihira, Langmuir, 13, 3261 (1997).

7) T. Ishida, S. Yamamoto, M. Motomatsu, W. Mizutani, H. Tokumoto, H. Hokari, H. Azehara, M. Fujihira, and I. Kojima, Jpn. J. Appl. Phys., 36, 3909 (1997).

8) H. Azehara, H. Hokari, U. Akiba, T. Ishida, W.
Mizutani, H. Tokumoto, and M. Fujihira, to be submitted.

9) C. A. Widrig, C. Chung, and M. D. Porter, J. Electroanal. Chem., 310, 335 (1991).

10) S. Arnold, Z. Q. Feng, T. Kakiuchi, W. Knoll, and K. Niki, J. Electroanal. Chem., 438, 91 (1997).

11) S. Imabayashi, D. Hobara, T. Kakiuchi, and W. Knoll, Langmuir, 13, 4502 (1997).

12) D. Hobara, M. Ota, S. Imabayashi, K. Niki, and T. Kakiuchi, J. Electroanal. Chem., 444, 113 (1998).

13) H. A. Biebuyck, C. D. Bain, and G. M. Whitesides, Langmuir, 10, 1825 (1994).

14) J. Clavilier, R. Faure, G. Guinet, and R. Durand, J. Electroanal. Chem., 107, 205 (1980).

15) I. Taniguchi, S. Yoshimoto, and K. Nishiyama, Chem. Lett., 1997, 353.

16) R. Yamada and K. Uosaki, Langmuir, 13, 5218 (1997).

17) G. E. Poirier and E. D. Pylant, Science, 272, 1145 (1996). 